\title{
Influence of commutation conditions on steady states in matrix converter*
}

\author{
DariUSZ Borkowski, TADEUSZ J. SOBCZYK \\ Institute on Electromechanical Energy Conversion \\ Cracow University of Technology \\ Warszawska 24, 31-155 Kraków, Poland \\ e-mail:dborkowski@pk.edu.pl, pesobczy@cyf-kr.edu.pl
}

(Received: 02.07.2010, revised: 02.12.2010)

\begin{abstract}
The paper investigates a significant influence of transients on steady states in a matrix converter with the one-periodic control strategy. Proposed controller can be used as an interconnection device within a power system for a power flow control. However, the presence of inductances in external systems has the significant influence on steady state of a matrix converter operation. The special current injection method has been developed to ensure a proper operation of a matrix converter. Presented analysis of steady states is carried out in a frequency domain using the harmonic balance method. Obtained numerical results, which are confirmed by a time domain analysis, prove the effectiveness of the proposed method.
\end{abstract}

Key words: matrix converter, power flow control, harmonic balance method

\section{Introduction}

Matrix converter on account of its universality has been investigated by research institutes in the whole world [1]. It can be used as a component of a FACTS unit, which allows to control power flow in a transmission line [2]. One of its most interesting application, is to use it as a power network interconnection device to avoid power flow oscillations resulting from disturbances in a power system. It is of the fundamental importance from a dynamic stability point of view. This application requires a dedicated control strategy, which ensures appropriate conditions. They seem to be fulfilled with the one-periodic control strategy, initiated and developed at the Institute of Electromechanical Energy Conversion at Cracow University of Technology [3]. This control strategy has some important advantages such as: high dynamic and control precision, limited amount of harmonics in input and output signals, the low sampling frequency [4] What is more, the voltage transfer ratio can be increased up to $98.8 \%$ by using two coupling transformers converting 3 phase system into 12 [5].

*This is extended version of a paper which was presented at the 21st Symposium on Electromagnetic Phenomena in Nonlinear Circuits, Essen-Dortmund, 29.06-02.07, 2010. 
Because of frequency analysis of that kind of units, description of the controller in frequency domain is desirable. It allows to determine analytical relations between parameters for particular harmonics (e.g. transfer ratio factor) and obtain steady state characteristics as a direct result of calculations [5]. Furthermore, it can be proved that voltages and currents on both sides of the converter are symmetrical [6].

\section{Analysis of a power flow controller in frequency domain}

In the analysis bidirectional switching elements are modelled as ideal (switching on and off in an arbitrary time instant) and represented by a set of conductances with step-wise variable parameters according to state functions (Fig. 1). To change a phase of output voltages it is enough to shift all state functions by a given time interval $T_{0}$. Thus, the voltage phase control is simple and easy to implement.

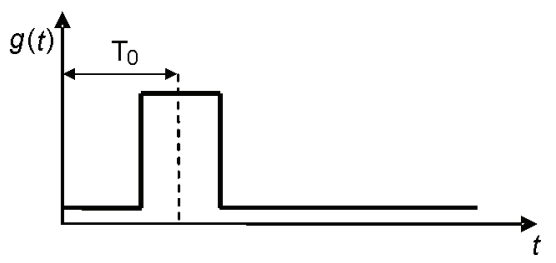

Fig. 1. An exemplary state function

To analyze a matrix converter in a transmission line external circuits have to be added in the form of symmetrical voltage sources $\left(\mathbf{e}_{\mathbf{i}}, \mathbf{e}_{\mathbf{o}}\right)$ with symmetrical internal impedances $\left(\mathbf{Z}_{\mathbf{i}}, \mathbf{Z}_{\mathbf{0}}\right)$ on both sides (Fig. 2).

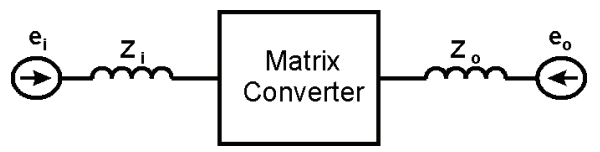

Fig. 2. A simplified scheme of matrix converter in a transmission line

Due to Fourier series and multiphase symmetrical components approach the system can be described by a linear infinite set of equations, which relate Fourier coefficients of currents, voltage sources, impedances and conductances [5].

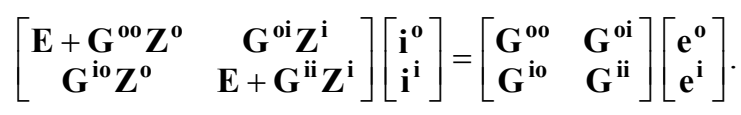

These equations determine the harmonics of currents with frequencies depending on the number of converter phases $N$ and a line frequency $f$ (for $p \in\{0, \pm 1, \pm 2, \ldots\}$ ).

$$
\mathrm{f}_{\mathrm{k}}=\mathrm{f} *|1+2 \mathrm{pN}| .
$$

The equations can be also used to predict quantitatively the currents in a transmission line and in consequence the power transferred for both voltage sources. For this, the dimension of 
equations have to be reduced to the finite one, but sufficiently high to obtain satisfactory results.

\section{Transient phenomena}

Testing calculations show that the presence of inductances in external systems has the significant influence on steady state of matrix converter operation. Fig. 3a shows RMS of line current harmonics with respect to the $\mathrm{k}_{\mathrm{XR}}$ factor for the constant absolute value of impedances. It is expected that the main harmonic should be constant (dashed line). In fact it isn't. It strongly depends on the $\mathrm{k}_{\mathrm{XR}}$ factor. What is more, this phenomenon is increased with a number of phases $N$ (Fig. 3b).

a)

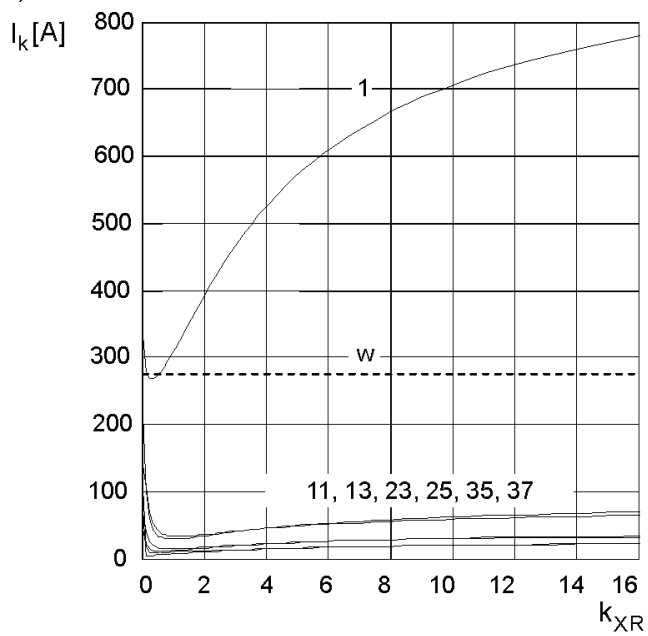

b)

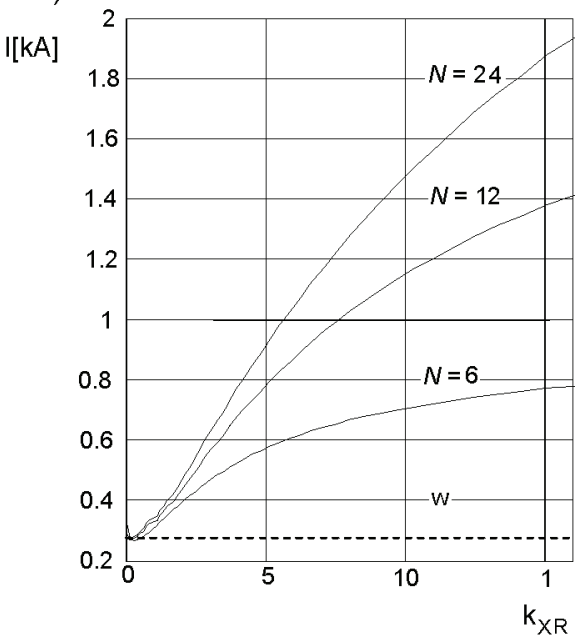

Fig. 3. The RMS of: a) harmonics of line current $(N=6)$; b) main harmonic of line currents for different phase number $(N=6,12,24)$, with respect to $\mathrm{k}_{\mathrm{XR}}$ factor for constant absolute value of impedances

It is caused by transient states of external systems. The current value is enlarged with each commutation step due to commutation conditions (defined by the $\mathbf{K}$ matrix) and instant values of currents just before the commutation $\mathbf{i}\left(0 \_\right)$.

$$
\mathbf{i}\left(0_{+}\right)=\mathbf{K} * \mathbf{i}\left(0_{-}\right) \quad \text { where }: \quad \mathbf{K}=\frac{1}{2}\left[\begin{array}{cccc}
1 & & & 1 \\
1 & 1 & & \\
& & \ddots & \\
& & 1 & 1
\end{array}\right] .
$$

This is clearly visible in the Fig. 4. Finally, the waveform of the steady state current signal is constituted of transients which increase pointedly the RMS value. 


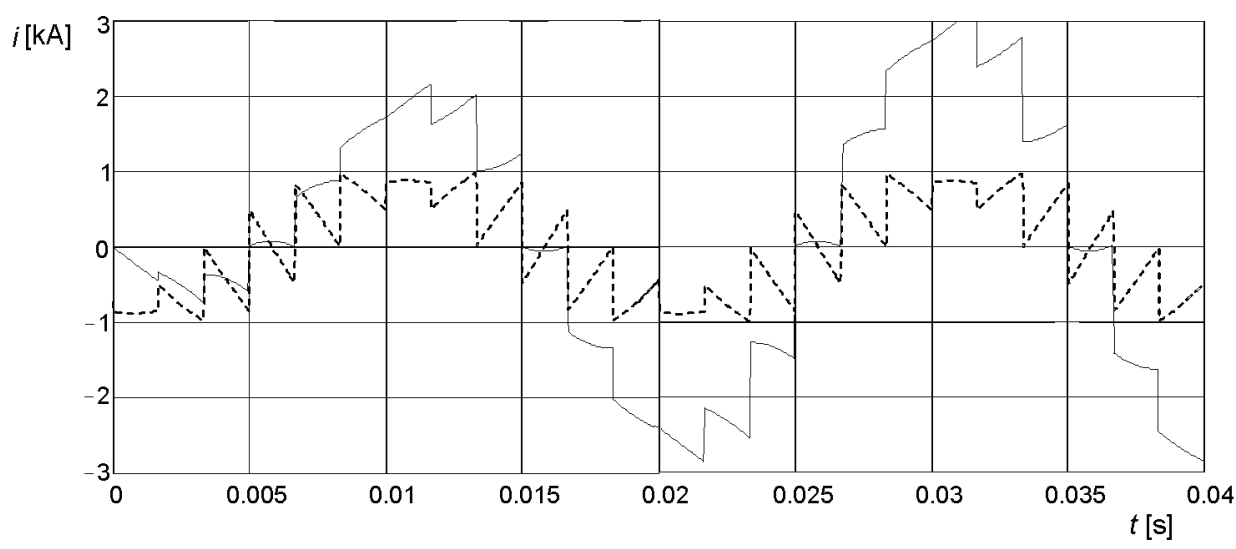

Fig. 4. Current waveforms in resistive (dashed line), inductance (full line) character of external system for six phase matrix converter

\section{Current injection method}

This negative influence of the commutation on converter operation could be eliminated by changing the commutation conditions. The key is to obtain the unitary commutation matrix $\mathbf{K}_{\mathrm{p}}$ (4), what may be done by the special strategy of current impulse injections $\mathbf{i}_{\mathrm{C}}$ into switches in commutation moments (5).

$$
\begin{gathered}
\mathbf{K}_{\mathbf{P}} * \mathbf{i}\left(\mathbf{0}_{-}\right)=(\mathbf{K}+\mathbf{C}) * \mathbf{i}\left(\mathbf{0}_{-}\right), \\
\mathbf{i}_{\mathbf{C}}=\mathbf{C} * \mathbf{i}\left(\mathbf{0}_{-}\right) .
\end{gathered}
$$

The principle of the impulse modulation may be defined by the formula:

$$
i_{\mathrm{C}}= \pm \mathrm{I}_{\mathrm{C}} \sin \left[\frac{\pi}{\mathrm{N}}\left(l-2 r+\frac{5}{2}\right)\right] \text {, }
$$

where: $\mathbf{I}_{\mathrm{C}}$ - amplitude of impulse envelope, $r$ - phase number, $l$ - commutation step number, \pm- sign dependent on injection side.

Exemplary current impulses for a 6-phase system are shown in Fig. 5. It should be emphasized that the mean value of all impulses injected in each commutation step is equal zero. So, we don't need to add any extra energy to the matrix converter. The energy, in the form of current impulses, is transfer between specific switches in each commutation step.

To introduce this methodology into presented equations (1) current impulses functions have to be describe in frequency domain using Fourier series (7).

$$
i_{C}(t)=\sum_{k=-\infty}^{\infty} \mathrm{B}_{\mathrm{k}} \mathrm{e}^{\mathrm{jk} \Omega \mathrm{t}}
$$

An exemplary current impulses function modeled in frequency domain and corresponding Fourier coefficient are presented in Fig. 6. 

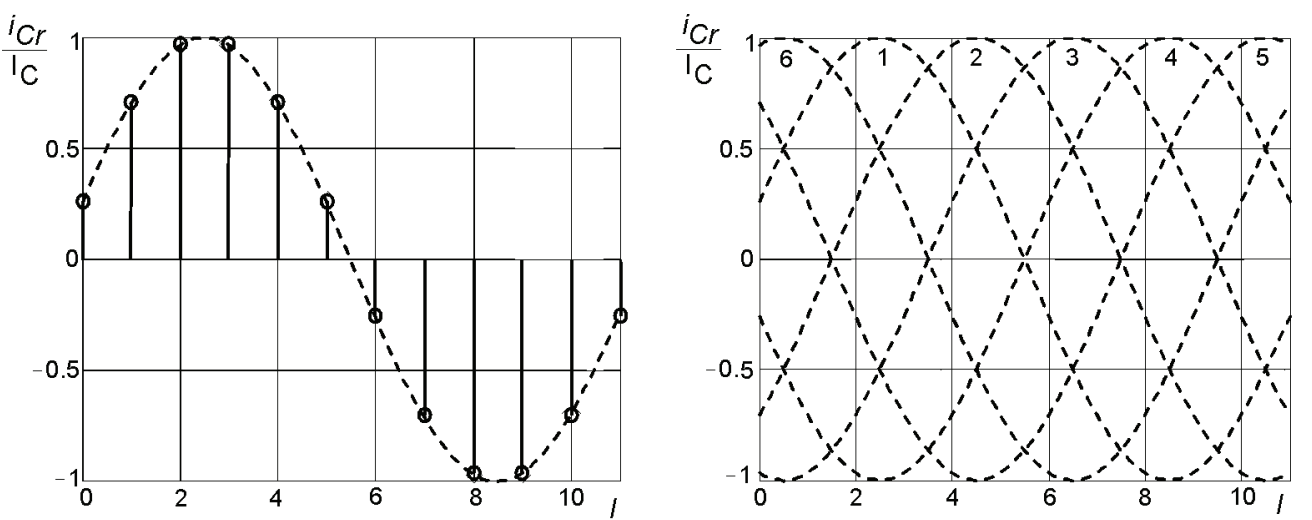

Fig. 5. Current impulses of 6-phase system for the first phase (on the left) and its envelopes (on the right)
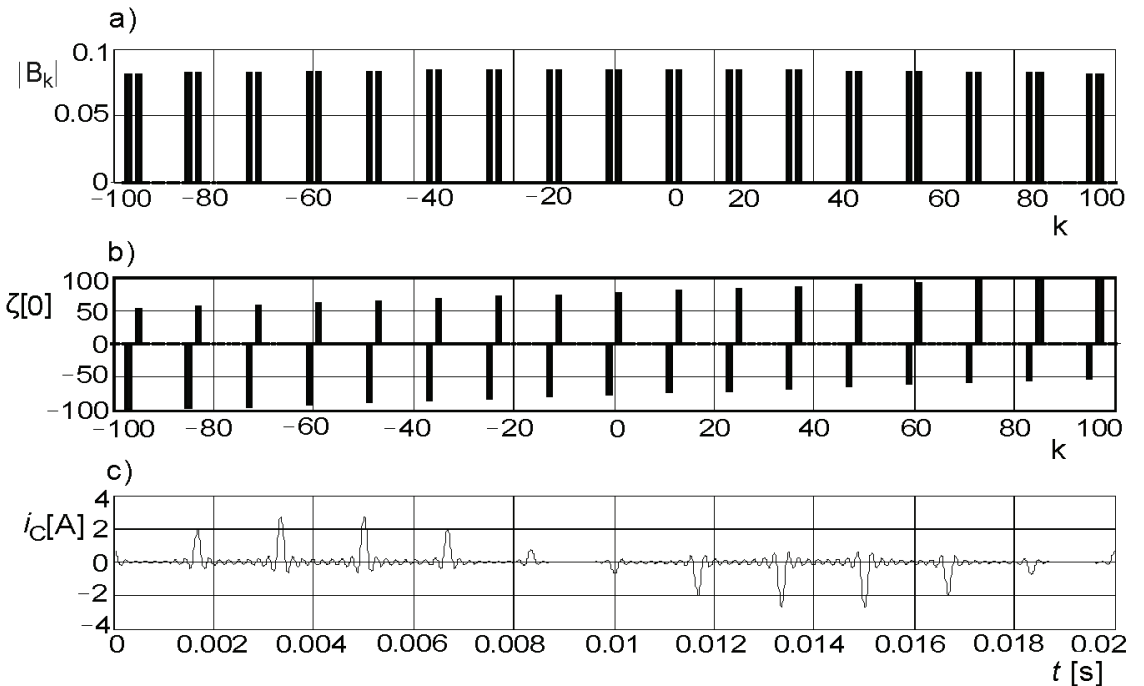

Fig. 6. Current impulses function modeled by Fourier series (c) and corresponding Fourier coefficients, its rms values (a) and phases (b)

The Fourier coefficients of current impulses function are introduce into description according to the formula:

$$
\left[\begin{array}{cc}
\mathbf{E}+\mathbf{G}^{\mathbf{0}} \mathbf{Z}^{\mathbf{0}} & \mathbf{G}^{\mathbf{0 i}} \mathbf{Z}^{\mathbf{i}} \\
\mathbf{G}^{\mathbf{i}} \mathbf{Z}^{\mathbf{0}} & \mathbf{E}+\mathbf{G}^{\mathrm{ii}} \mathbf{Z}^{\mathbf{i}}
\end{array}\right]\left[\begin{array}{l}
\mathbf{i}^{\mathbf{0}} \\
\mathbf{i}^{\mathbf{i}}
\end{array}\right]=\left[\begin{array}{ll}
\mathbf{G}^{\mathbf{0 0}} & \mathbf{G}^{\mathbf{0 i}} \\
\mathbf{G}^{\mathbf{i}} & \mathbf{G}^{\mathbf{i i}}
\end{array}\right]\left[\begin{array}{l}
\mathbf{e}^{\mathbf{0}} \\
\mathbf{e}^{\mathbf{i}}
\end{array}\right]+\left[\begin{array}{l}
\mathbf{B}^{\mathbf{0}} \\
\mathbf{B}^{\mathbf{i}}
\end{array}\right]
$$

where: $\mathbf{B}^{0}, \mathbf{B}^{\mathrm{i}}$ - vector representation of Fourier series coefficients of the impulse sequence function.

The numerical calculation results of the linear equations set (8) are verified using a time domain modeling by a comparison of currents waveform (Fig. 7). The proper choice of para- 
meters and transformations of the equation set improve its condition and allow to obtain the numerical convergence.
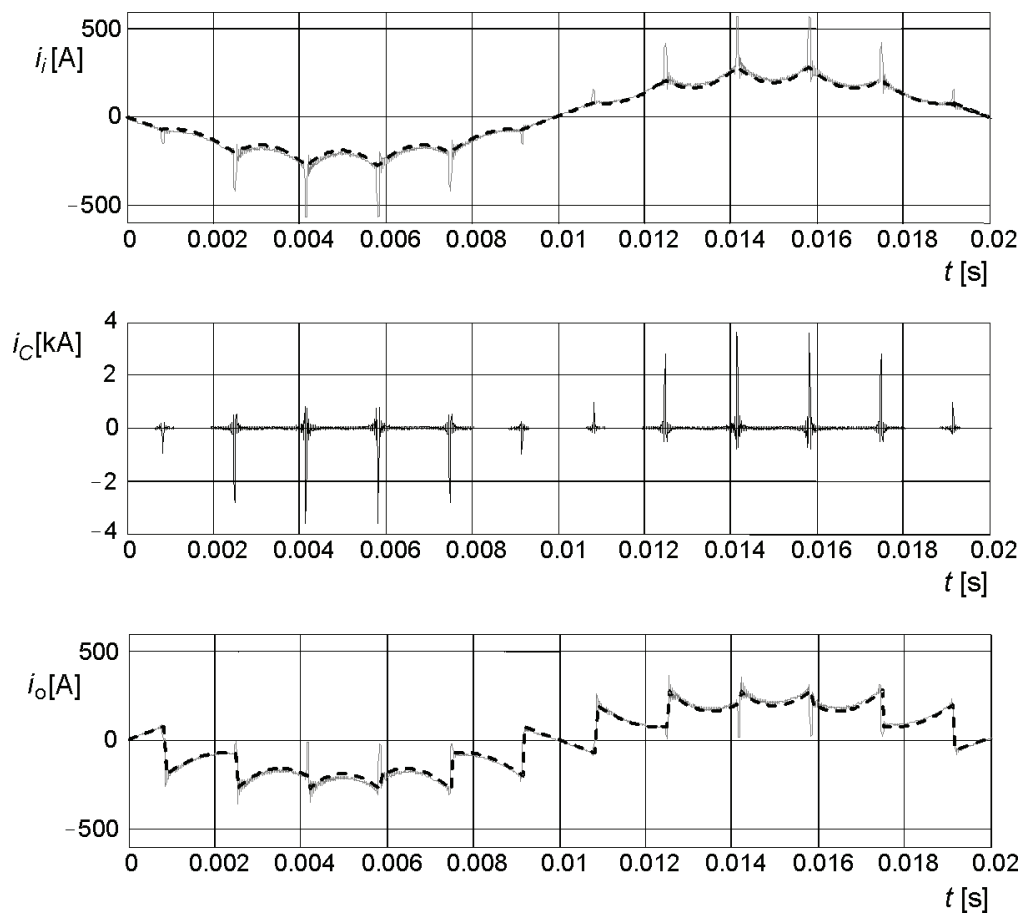

Fig. 7. Input (first plot) and output (third plot) current waveforms obtained from: frequency analysis (full lines) and time analysis (dashed lines). Current impulses (second plot)

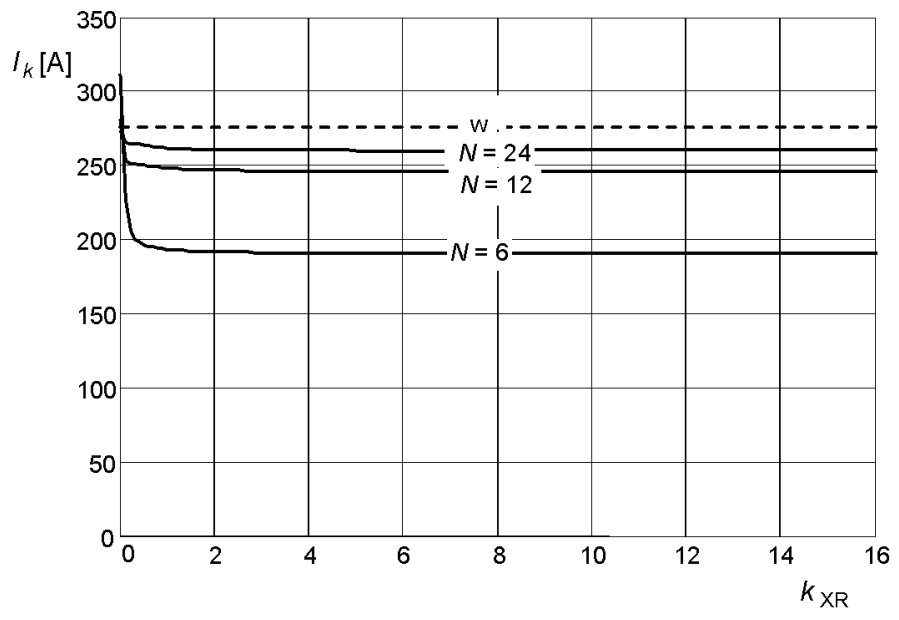

Fig. 8. The RMS of line currents main harmonic for different phase number $(N=6,12,24)$, with respect to $k_{\mathrm{XR}}$ factor for constant absolute value of impedances in the case of applying the current injection method 
Presented current injection methodology allows to change commutation conditions and obtain proper operation of the matrix converter. Fig. 8 presents RMS of line current harmonics with respect to the $\mathrm{k}_{\mathrm{XR}}$ factor for the constant absolute value of impedances, just like Fig. $3 \mathrm{~b}$. But here the current injection methodology is introduced what ensure proper RMS value of the main harmonic. Main harmonics still slightly differ from the model value (dashed line) what is caused by parasitic current. This parasitic current appear because the voltage transfer factor is less than one. However, the value of this current could be reduced by increasing the voltage transfer factor up to $98.8 \%$ for $N=24$ [5].

\section{Conclusions}

Presented analysis deals with a negative influence of the commutation process on a converter operation by the special current injection method. This methodology may be realized without adding any extra energy what is an important feature and its effectiveness is proved by numerical calculations. Proposed method defines proper commutation conditions which should be implemented by the special technical realization.

Furthermore, presented methodology, basing on symmetrical components approach and Fourier series, may be also used for modelling switching power losses by introducing resistance impulses functions [7]. Therefore, this is valuable and promising approach which may be used in various applications.

\section{References}

[1] Wheeler P.W., Matrix Converters: A Technology Review. IEEE Transactions On Industrial Electronics 49(2): 276-288 (2002).

[2] Yong Hua Song, Johns A.T., Flexible ac transmission system (FACTS). The Institution of Electrical Engineers (1999).

[3] Sienko T., Sobczyk T.J., An algorithm of matrix converter control. Polish patent PL - 204643 (2003).

[4] Sienko T., Szczepanik J.,. Sobczyk T.J, Voltage Phase Controller for Power Systems. Proc. of 9th Int. Conf. Electrical Power Quality and Utilisation, pp. 1-6, Barcelona (2007).

[5] Sobczyk T.J., Borkowski D., Application of Matrix Converter for Power Flow Control in a Transmission Line. Proc. of Power Tech., Lausanne 138: 1823-1828 (2007).

[6] Sobczyk T.J., Voltage-current equations of power electronic matrix converter in frequency domain. Archives of Electrical Engineering LI(1): 105-114 (2002).

[7] Borkowski D. The Analysis of a Matrix Converter as an Interconnection Device in the Power System. PhD thesis (in Polish), Cracow (2010). 